\title{
3. Hyperthermia- for Cancer Therapy
}

\author{
Sunao EGAWA
}

Department of Radiation Therapy, National Cancer Center Hospital, Tokyo

The clinical application of hyperthermia to treatment of cancer has increased in recent years, and knowledge on its applicability and efficacy are increasing. The therapy is now conducted at many hospitals but the technical difficulties related to the device or temperature measurement still remain unsolved.

Protocols for combined use with radiotherapy or chemotherapy have not been fully established, and yet the progress is being steadily made in this area. The recent progress was supported by biological experiments to observe the effects of high temperature more than $42^{\circ} \mathrm{C}$ on cell survival ratio, and development of devices applying physical energy capable of heating localized area have become clinically available.

\section{FUNDAMENTAL ASPECTS}

(1) Heating Equipment

Heating devices come in two categories; those for heating superifical tumors and those for heating tumors at depth. As the latter device is not capable of heating the tumor alone, the tumor and its surrounding tissue must be heated.

The current devices can certainly raise the temperature of superficial tumor which should be at least $42.5^{\circ} \mathrm{C}$. The frequency of microwave is determined depending on the size and depth of the tumor, but such conditions are not necessarily selectable freely at present. Rather, the types of tumor are selected for treatment according to the specifications of the device. Thus, the need for selecting a device with the widest scope of applicability arises at a hospital.

Attempts at directly heating the tumor by penetrating the heating needle into the tumor are being made. This is called the interstitial heating method and uses microwave antenna, RF electrode needles, and ferromagnetic needles. Combined with interstitial radiothreapy, the method will be most effective procedures so far.

\section{(2) Biology of Hyperthermia Therapy}

When normal cells and cancer are compared regarding thermosensitivity, the latter seems to have somewhat higher sensitivity, but not significantly. The environment surrounding cancer cells often has low $\mathrm{pH}$, poor blood flow, and low oxygen partial pressure. They are important factors for enhancing sensitivity for hyperthermia. The temperature needed for hyperthermia therapy is at least $42.5^{\circ} \mathrm{C}$, because the mechanism for inducing hyperthermic effects is believed to differ beneath and above this value. Therefore, the time needed to induce the identical effects above $42.5^{\circ} \mathrm{C}$ is less: namely, if the temperature is raised by $1{ }^{\circ} \mathrm{C}$, the time becomes $1 / 2$; while below $42.5^{\circ} \mathrm{C}$, the time needed becomes 4 times longer with $1^{\circ} \mathrm{C}$ temperature decrease. Thermal dose which normalize the amount of the effect at different temperature to $42.5^{\circ} \mathrm{C}$ or $43^{\circ} \mathrm{C}$ was proposed. In the hyperthermia therapy, a session generally lasts for 1 hours. And yet, very high temperature is injurious to the normal tissue. The temperature of cancer, for instance region in the center, rises as high as $50^{\circ} \mathrm{C}$, but this dose not pose problems.

There are often cases involving a tumor where the blood flow is ample at the periphery but nonexistent at the center because of necrosis. When heated, the temperature rises rapidly at the center but not at the periphery as the heat escapes to other regions borne the blood flow. This results in distribution of non-uniform temperature within a tumor. In the treatment of a tumor, the temperature close to the tumor is thus used for a reference to see if it is above or beneath $42.5^{\circ} \mathrm{C}$. Therefore, with a thermometer placed inside a tumor, the observation of temperature distribution is important in clinical medicine. Although its routine application is not feasible, effects are being made to learn the temperature distribution as much as possible.

Problems of thermotorelance relating to the 
production of heat shock protein are widely studied but still fully unsolved.

\section{CLINICAL EXPERIENCES}

The usual practice is to combine it with radiation therapy or chemotherapy. As many basic researches are being performed on enhanced effects of hyperthermia by irradiation and drugs, protocols for combined therapies have been prepared. The combination with radiotherapy is often studied as both treatments can be applied to localized lesions. In many cases, irradiation is given 5 times a week with 1 to 2 sessions of hyperthermia, or patients are given irradiation usually followed by hyperthermia treatment on the same days twice a week. A heat application lasts for 1 hour at a time and repeated 7 to 8 times or sometimes 10 times. Temperature monitoring is necessary, and the temperature profile of the tumor should perferably be drawn by measuring at least one point inside the tumor or while placing in and drawing out the thermometer if possible. The temperature sensor should be made of a material which is not affected by the physical energy employed.

In the case of a massive tumor, the cencer necrotic focus becomes extended by heating, and is often shown as low absorbent region in $\mathrm{X}$-ray CT. Though the reduction of tumor size in such cases is not prominent, we often find no residual tumor cells present upon surgical extripation of tumor. It is one of the controversial points in judging the therapetic effect on tumor.

When combined use with radiotherapy is compared to radiotherapy alone, approximately 2 fold increase is observed in cure rate of localized lesion, and the effectiveness in the treatment of malignant melanoma and adenocarcinoma which are less sensitive to radiation is evident. In the patients with relapse after irradiation, re-irradiation and heating were observed to enhance therapeutic effect.

Our recent results of hyperthermia and radiation therapy for superficial and deep seated tumors are as follows. Local defect (CR+PR) for superficial tumors was $60 \%$. For deep seated tumors it was $23 \%$. The results could be reinterprete applying the criteria for local effect in histological examination, X-CT findings. Among 48 cases of deep seated tumors thus analyzed, 32 cases were effective.

The evaluation of the hyperthermia should be performed with well designed controlled trial. So far, the results were interpreted by the comparison with historical control at best. Because non randamized trial showed promising results, further verification by the controlled traials are strongly needed.

Combined use with chemotherapy appears promising. With a drug capable of potentiating thermal effects at relatively low temperatures $\left(40^{\circ} \mathrm{C}-42^{\circ} \mathrm{C}\right.$ or thereabout), hyperthermia therapy can be applied further.

Systemic heating is a variation of hyperthermia therapy. Extracorporeal circulation system is used to remove blood from femoral artery, warm it and then to return to femoral vein. The treatment is conducted under rigorous supervision and only a limited number of institutions use this sytem.

\section{CONCLUSION}

Hyperthermia in cancer therapy is still in the stage of development. Urgent problems to be solved are the development of non-invasive thermometry, interpretation of the relation of thermotolerance and heat shock protein, acumulation of the results for hyperthermia of deep seated tumors, and conduction of the well designed controled trial in order to evalnate the effect of hyperthermia.

\section{REFERENCES}

1) Egawa, S: Cancer and Hyperthermia therapy. Shinohara Shuppan, 1987. (in Japanese)

2) Sugahara, $T$ and Abe, M: hyperthermmia, a New Modality for Treating Cancer. Magbros Shuppan, 1984. (in Japanese)

3) Egawa S, Tsukiyama, I, Akine $Y$, et al. Results of local effect on Malignant tumors treated with combined radiotherapy and hyperthermia, Jpn. J. Hyperthermic Oncology $3: 21-26,1987$. (in Japanese)

4) Egawa S, Tsukiyama I, Kajiura $Y$, et al. Hyperthermai-Therapy for Cancer, Asian Medical Journal 30: 20, 1987. 